\title{
Radio continuum spectra of galaxies in the Virgo cluster region ${ }^{\star} \star \star$
}

\author{
B. Vollmer ${ }^{1,2}$, M. Thierbach ${ }^{2}$, and R. Wielebinski ${ }^{2}$ \\ ${ }^{1}$ CDS, Observatoire astronomique de Strasbourg, UMR 7550, 11 rue de l'université, 67000 Strasbourg, France \\ 2 Max-Planck-Institut für Radioastronomie, Auf dem Hügel 69, 53121 Bonn, Germany
}

Received 28 November 2003 / Accepted 21 December 2003

\begin{abstract}
New radio continuum observations of galaxies in the Virgo cluster region at 4.85, 8.6, and $10.55 \mathrm{GHz}$ are presented. These observations are combined with existing measurements at 1.4 and $0.325 \mathrm{GHz}$. The sample includes 81 galaxies where spectra with more than two frequencies could be derived. Galaxies that show a radio-FIR excess exhibit central activity (HII, LINER, AGN). The four Virgo galaxies with the highest absolute radio excess are found within $2^{\circ}$ of the center of the cluster. Galaxies showing flat radio spectra also host active centers. There is no clear trend between the spectral index and the galaxy's distance to the cluster center.
\end{abstract}

Key words. galaxies: clusters: individual: Virgo - galaxies: evolution - galaxies: magnetic fields - radio continuum: galaxies

\section{Introduction}

A galaxy cluster is an ideal laboratory to study the influence of the galaxies' environment on its appearance and/or evolution. It is a well established fact that spiral galaxies in clusters have less atomic gas than isolated spirals of the same morphological type and same optical diameter, i.e. they are HI deficient (Chamaraux et al. 1980; Bothun et al. 1982; Giovanelli \& Haynes 1985; Gavazzi 1987, 1989). There are mainly two kinds of mechanisms that are able to cause the removal of the atomic gas: (i) tidal interactions or (ii) the interaction of the interstellar medium (ISM) with the hot intracluster medium (ICM). The mapping of the gas content of spiral galaxies in the Virgo cluster (Cayatte et al. 1990, 1994) showed that the HI disk sizes of cluster spirals are considerably reduced. In addition, galaxies with a symmetric optical disk that have an asymmetric HI gas distribution are quite frequent in the cluster core. These observational results indicate that gas removal due to the rapid motion of the galaxy within the ICM (ram pressure stripping; Gunn \& Gott 1972) is responsible for the HI deficiency and the distorted gas disks of the cluster spirals.

During the phase of active ram pressure stripping the interstellar matter (ISM), which is located in the outer disk,

Send offprint requests to: $\mathrm{B}$. Vollmer,

e-mail: bvollmer@astro.u-strasbg.fr

* Figure 3 is only available in electronic form at http://www.edpsciences.org

$\star \star$ Table 3 is only available in electronic form at the CDS via anonymous ftp to cdsarc.u-strasbg.fr $(130.79 .128 .5)$ or via http://cdsweb.u-strasbg.fr/cgi-bin/qcat?J/A+A/418/1 is pushed to smaller radii where it is compressed (Vollmer et al. 2001). This effect is more important for edge-on stripping than for face-on stripping. Since the magnetic field is frozen into the ISM, the magnetic field $B$ becomes compressed too and its strength is enhanced (Otmianowka-Mazur \& Vollmer 2003). The radio continuum emission due to relativistic particles gyrating around the magnetic fields is proportional to $B^{2-4}$ depending on whether there is equipartition between the relativistic electrons and the magnetic field. One might thus expect an enhancement of the radio continuum emission of spiral galaxies that undergo active ram pressure stripping. This enhancement should be detectable as a radio excess in the well studied radio-FIR correlation (see, e.g., de Jong et al. 1985; Wunderlich et al. 1987; Niklas 1997).

Furthermore, one might speculate that ram pressure stripping triggers nuclear activity. Since radio spectra of centrally active galaxies (HII, LINER, AGN) are generally flatter than the synchrotron spectra of normal galaxies (mean spectral index $\alpha \sim-0.7$, Israel \& van der Hulst 1983), one might expect flatter radio spectra close to the cluster center.

Gavazzi \& Boselli (1999a) studied the radio luminosity function of Virgo cluster galaxies for early and late type galaxies separately. They found that late type galaxies develop radio sources with a probability proportional to their optical luminosity, independently of their detailed Hubble type. In a second paper Gavazzi \& Boselli (1999b) compared the radio luminosity functions of galaxies in different clusters to those of isolated galaxies. They concluded that the radio luminosity function of Virgo cluster galaxies is consistent with that of isolated galaxies, whereas the Coma cluster galaxies show an excess of radio emissivity. 
Niklas et al. (1995) investigated the behaviour of the radio-FIR correlation and the radio spectra in the Virgo cluster. They claim that galaxies that show an excess of radio emission are located close to the cluster center, whereas the spectra do not seem to be affected by the location of the galaxies in the cluster.

In this article we extend the galaxy sample of Niklas et al. (1995) by adding observations at $8.6 \mathrm{GHz}$ and enlarging the sample to 81 galaxies, which were observed at $10.55,8.6$, $4.85 \mathrm{GHz}$ with the Effelsberg 100-m telescope. Our data is complemented by $1.4 \mathrm{GHz}$ flux densities from the NVSS where advantage is taken of the NVSS images to check for source confusion and to estimate the correct source flux.

The sample and the observations are presented in Sects. 2 and 3. We show the results in Sect. 4 and investigate the radioFIR correlation in Sect. 5. The distribution of the radio spectra are studied in Sect. 6 followed by the discussion and our conclusions (Sect. 7).

\section{The sample}

Gavazzi \& Boselli (1999a) derived the radio luminosity function of the Virgo cluster by cross-correlating optical data of the Virgo Cluster Catalogue (VCC) (Binggeli et al. 1985) with NVSS radio survey carried out at the VLA at $1.4 \mathrm{GHz}$ (Condon et al. 1998). The 180 positive radio-optical matches that they found build the basis of our survey. We restrict ourselves to radio sources with $1.4 \mathrm{GHz}$ flux densities greater than $10 \mathrm{mJy}$, which corresponds to a flux density at $10.5 \mathrm{GHz}$ of $2 \mathrm{mJy}$, assuming a spectral index $\alpha=-0.8$, where

$S \propto v^{\alpha}$

( $S$ is the radio flux density and $v$ the frequency). We thus observed 81 galaxies of various Hubble types. Their distribution on the sky is shown in Fig. 1. The number of galaxies of different types are listed in Table 1. As expected, the sample is clearly dominated by late type galaxies $(\mathrm{Sc} / \mathrm{Scd})$ with a high star formation rate. There are also 10 dwarf galaxies in our sample. We took the $\lambda 20,50 \mathrm{~cm}$ flux densities and the IRAS FIR fluxes from the Goldmine database ${ }^{1}$ and the position angle of the galactic disks from LEDA ${ }^{2}$. The FIR flux is calculated according to Helou et al. (1988) in the following way:

$$
F I R=2.58 S_{60_{\mu}}+S_{100_{\mu}}
$$

where $S_{60_{\mu}}$ is the flux at $60 \mu \mathrm{m}$ and $S_{100_{\mu}}$ the flux at $100 \mu \mathrm{m}$.

Figure 2 shows the number of galaxies as a function of the corrected $B$ band magnitude in bins of $0.5 \mathrm{mag}$. For comparison, the complete sample of Virgo galaxies brighter than $m_{B}=18$ (Gavazzi \& Boselli 1999a) is also shown. Most of the missing galaxies in our sample compared to that of Gavazzi \& Boselli (1999a) have photographic magnitudes around $m_{B}=12$ and $m_{B}=14$.

\footnotetext{
${ }^{1}$ Operated by the University of Milano-Bicocca (see Gavazzi et al. 2003, A\&A, 400, 451).

${ }^{2}$ HyperLeda is an extragalactic database located at http://leda.univ-lyon $1 . f r /$
}

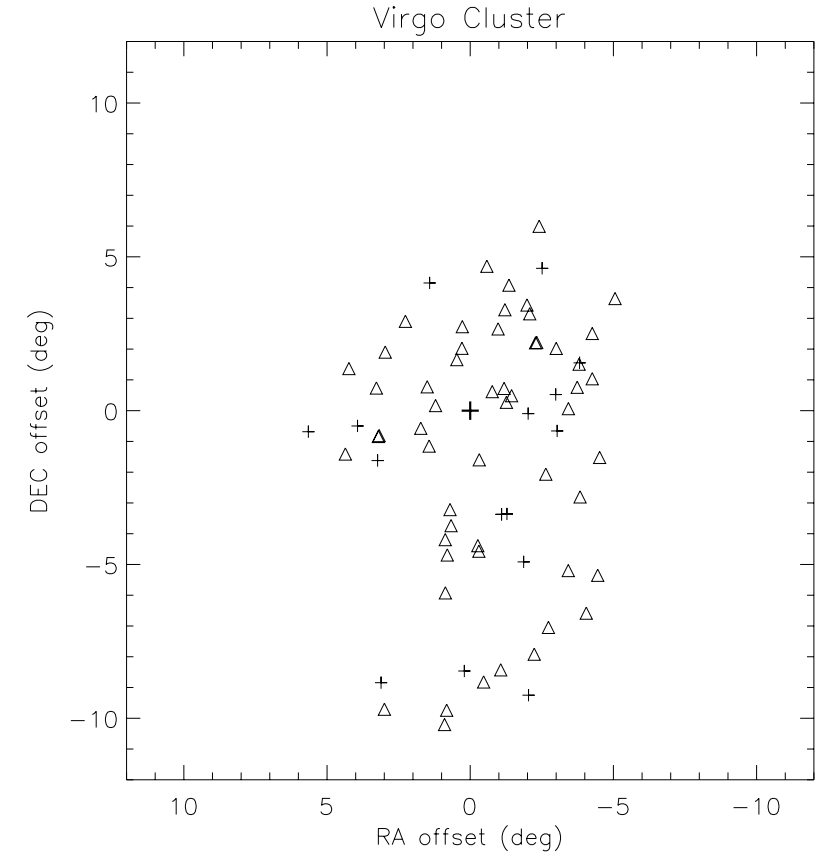

Fig. 1. Distribution of the observed galaxies. Triangles: Virgo galaxies. Crosses: background galaxies. Thick cross: M 87.

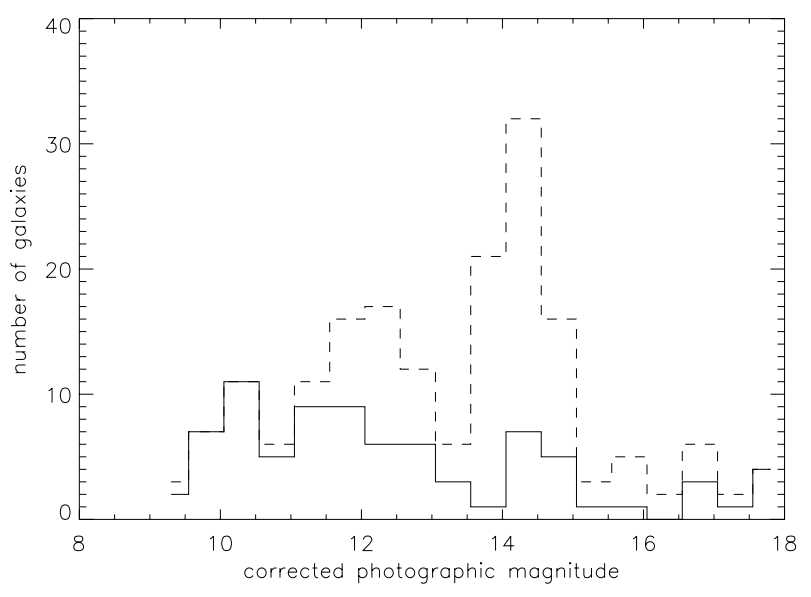

Fig. 2. Number of galaxies as a function of the corrected $B$ band magnitude in bins of $0.5 \mathrm{mag}$. Solid: our sample. Dashed: complete sample of Virgo galaxies brighter than $m_{B}=18$ (Gavazzi \& Boselli 1999a).

Table 1. The galaxy sample: number of galaxies per Hubble type.

\begin{tabular}{|c|c|c|c|c|c|c|c|}
\hline$\overline{\overline{\mathrm{E}} / \mathrm{S} 0}$ & 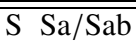 & $\overline{\mathrm{Sb} / \mathrm{Sbc}}$ & $\overline{\mathrm{Sc} / \mathrm{Scd}}$ & "Sm/Im & $\overline{\mathrm{Pec}}$ & & $\overline{\mathrm{BCD}}$ \\
\hline 12 & 10 & 10 & 33 & 2 & 2 & 8 & 2 \\
\hline
\end{tabular}

\section{Observations}

Our sample of 81 galaxies was observed in November 2000 , January 2001, August/September/November 2002, and April/ June 2003 with the 100-m Effelsberg telescope at 4.85, 8.6, and $10.55 \mathrm{GHz}(6.0,3.6$, and $2.8 \mathrm{~cm})$. The frequency, $H P B W$, system temperature, bandwidth, and number of beams are listed in Table 2. The existence of several beams at 4.85 and $10.55 \mathrm{GHz}$ permits one to subtract non-linear baselevels due to meteorologic conditions. The most sensitive receiver is the one at 
Table 2. Observations characteristics.

\begin{tabular}{ccccc}
\hline \hline frequency $(\mathrm{GHz})$ & $H P B W\left({ }^{\prime \prime}\right)$ & $T_{\text {sys }}(\mathrm{K})$ & bandwidth $(\mathrm{GHz})$ & beams \\
\hline 4.85 & 147 & 9 & 0.5 & 2 \\
8.6 & 85 & 4 & 1.2 & 1 \\
10.55 & 69 & 50 & 0.3 & 4 \\
\hline
\end{tabular}

8.6 GHz due to its wide bandwidth and low system temperature. Since it is a single beam receiver, perfect weather conditions are needed for observations at this frequency.

The galaxies were measured with cross-scans. The crossscans were centered on the radio center of the galaxies given by Gavazzi \& Boselli (1999a). The scanning directions were along the major and minor axis of the galaxies. The length of the cross-scans were $16^{\prime}, 15^{\prime}$, and $10^{\prime}$, the duration of the scan 24,30 , and $30 \mathrm{~s}$ at $4.85,8.6$, and $10.55 \mathrm{GHz}$ respectively. The number of cross-scans and thus the total integration time at a given frequency was calculated using the expected flux density extrapolated from the NVSS flux at $1.4 \mathrm{GHz}(\alpha=-0.8)$ and a $S / N$ of 5 . The calibration scale for the fluxes is that of Ott et al. (1994). For a correct calibration of the cross-scans and telescope pointing 3C 286 was observed frequently during the observations.

To derive the flux densities of the cross-scanned galaxies, Gaussians were fitted to the averaged scans in each direction. These fits give the peak flux value $S^{\text {peak }}$ and the half-power width $H P W^{\text {obs }}$ of the fitted Gaussian and the positional offset. The peak value of each scan direction was then corrected using the following formula to obtain the total flux density $S_{\text {tot }}$ :

$S_{\text {tot }}=S_{1}^{\text {peak }} \times S_{2}^{\text {peak }} \times\left(\frac{H P W_{1}^{\mathrm{obs}}}{H P B W}\right) \times\left(\frac{H P W_{2}^{\mathrm{obs}}}{H P B W}\right)$,

where $S_{i}^{\text {peak }}$ and $H P W_{i}^{\text {obs }}$ with $i=1,2$ are the flux densities and half-power widths along the major and minor axis of the galaxies. For the determination of the source extent, the $10.55 \mathrm{GHz}$ and $8.6 \mathrm{GHz}$ data are best suited, because they have the highest resolutions. Unfortunately, meteorological conditions and frequent source confusion did us not allow to derive reliable half-power widths from our data. Therefore, we used our peak fluxes and the half power widths at $1.4 \mathrm{GHz}$ given by Gavazzi \& Boselli (1999a).

Source confusion is a serious problem in the Virgo cluster region, especially at $4.85 \mathrm{GHz}$. Our advantage is that we have the NVSS $1.4 \mathrm{GHz}$ fluxes, where the correct source has been identified on the NVSS images (Gavazzi \& Boselli 1999a). Thus, it was possible to identify the confusing source, to subtract it, and to fit a Gaussian to the radio source belonging to the correct VCC source. Since Niklas et al. (1995) did not have the NVSS flux densities they could not decide which source was the correct one. Thus we had to re-observe several of their measured galaxies.

\section{Results}

The observed flux densities at 4.85, 8.6, and $10.55 \mathrm{GHz}$ are listed in Table 3. We have also included the measurements of Niklas et al. (1995), which are marked in boldface. The columns of Table 3 are: (1) VCC name of the galaxy; (2) right ascension in B1950 coordinates; (3) declination in B1950 coordinates; (4) projected distance from the Virgo cluster center (M 87); (5) Hubble type; (6) Virgo cluster membership based on radial velocities $(y / n)$; (7) flux density at $10.55 \mathrm{GHz}$; (8) flux density at $8.6 \mathrm{GHz}$; (9) flux density at $4.85 \mathrm{GHz}$; (10) flux density at $1.4 \mathrm{GHz}$; (11) flux density at $0.6 \mathrm{GHz}$; (12) FIR flux (see Eq. (2)); (13) spectral index $\alpha$ (see Eq. (1)). When we did not detect a galaxy at $4.85,8.6$, and $10.55 \mathrm{GHz}$ the upper limit of the flux density is $\sim 1 \mathrm{mJy}$ at $10.55 \mathrm{GHz}$.

We made linear fits in the $\log v-\log S_{v}$ plane using the method of the least absolute deviation. This method is less sensitive to outlying points in the spectrum and thus more robust than a $\chi^{2}$ fit. The obtained spectra are shown in Fig. 3. The spectral index $\alpha$ is plotted in the lower left corner of the boxes. We only fitted a spectrum when there are more than two data points.

Since the cross-scan method is less accurate than imaging of the sources, the spectra are noisier than those obtained by direct imaging. The main error is due to offsets in the Gaussian fits and imprecise position angles of the galaxies. In many cases the flux densities at $10.55 \mathrm{GHz}$ are lower than expected by our fitted spectrum. This might be due to the fact that this receiver is the least sensitive of the three receivers used. Moreover, the background subtraction using a second beam has the effect that the total flux might be underestimated.

\section{The radio-FIR correlation}

In Figs. $4 \mathrm{a}-\mathrm{d}$ and $\mathrm{f}$ we show the radio-FIR correlation for radio frequencies $10.55,8.6,4.85,1.4$, and $0.6 \mathrm{GHz}$ including all galaxies of the sample. The early type galaxies (E) of our sample are marked as triangles. Only one elliptical appears in Figs. 4a, b, because there are no FIR flux densities measured for the other Virgo early type galaxies. Thus, the sample we use here is made up of spiral (Sa-Im) and S0 galaxies except VCC0763. The least absolute deviation fits are also shown as solid lines whose slopes are plotted in the lower left corners of the boxes. The major outlying galaxies are labeled. In order to investigate if the radio excess of these outliers is located in the disk or in the center of the galaxies, we subtracted the FIRST 1.4 GHz flux densities for sources where the FIRST image shows a central point source (the FIRST resolution is $6^{\prime \prime}$ ). The radio-FIR correlation using these corrected $1.4 \mathrm{GHz}$ flux densities can be seen in Fig. 4e. Clearly, the radio excesses are due to central point sources, i.e. central activity (LINER, HII, AGN). The slopes of the radio-FIR correlation at 4.85 and $1.4 \mathrm{GHz}$ are consistent with those of samples of isolated galaxies (Wunderlich et al. 1987; Niklas 1997). At $10.55 \mathrm{GHz}$ our radio data is quite noisy, but when we remove the outlying points the slope is consistent with that found by Niklas et al. (1995) and Niklas (1997). The radio-FIR correlations at 8.6 and $0.6 \mathrm{GHz}$ fit also in this picture.

To investigate the location of the Virgo galaxies that show a strong absolute radio excess within the cluster, Fig. 5 shows the absolute radio excess at $4.85 \mathrm{GHz} S_{4.85 \mathrm{GHz}}-S_{\mathrm{FIR}}$ as a function of the projected distance to the cluster center (M 87). 

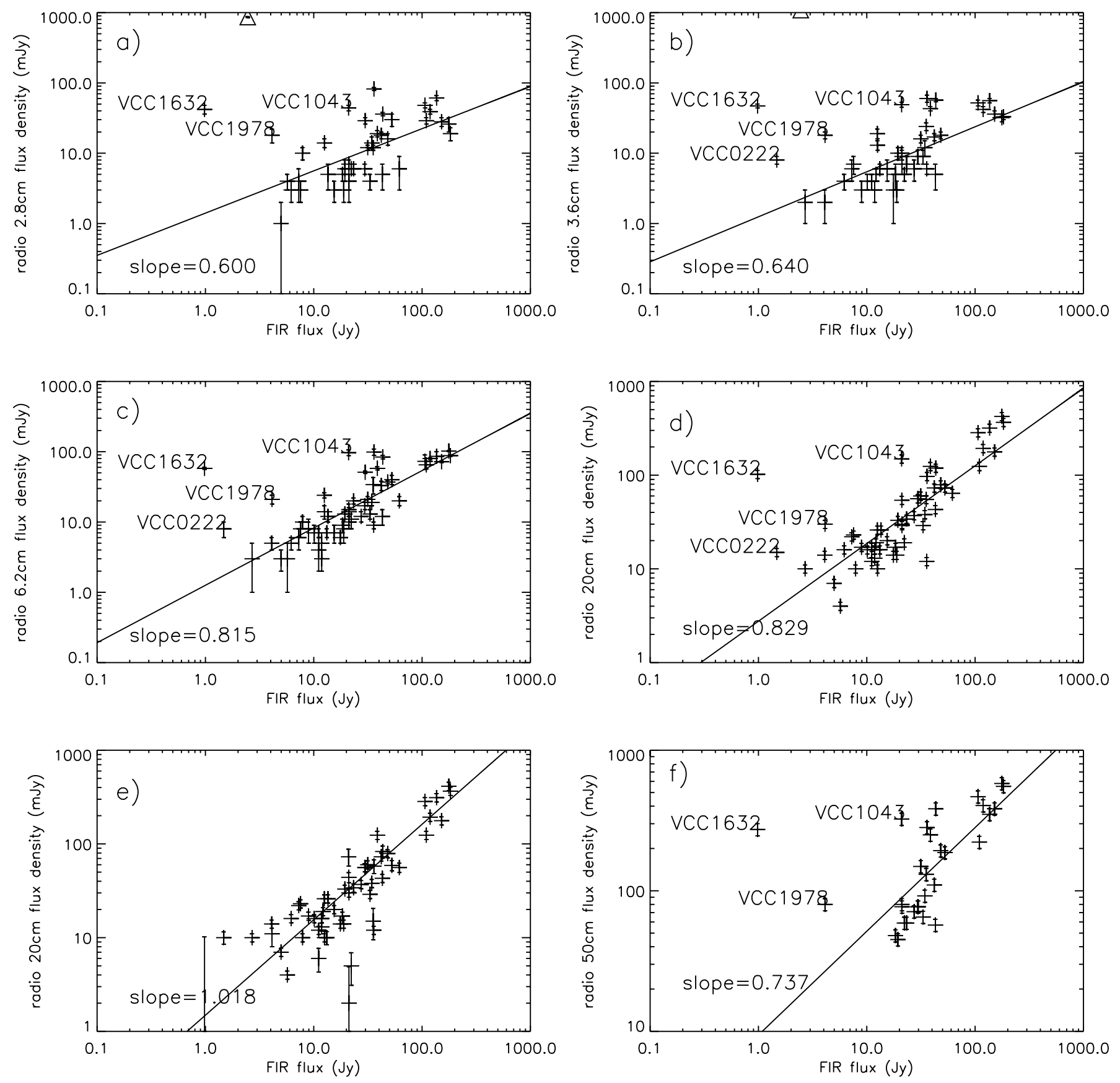

Fig. 4. Radio-FIR correlation: a) at $10.55 \mathrm{GHz} ; \mathbf{b})$ at $8.6 \mathrm{GHz}$; c) at $4.85 \mathrm{GHz}$; d) at $1.4 \mathrm{GHz}(\mathrm{NVSS})$; e) at $1.4 \mathrm{GHz}$ FIRST central point source flux densities subtracted; f) at $0.6 \mathrm{GHz}$.

The radio excess is defined as the difference between the observed and the expected radio flux from the radio-FIR correlation. All shown galaxies are classified as spiral or S0 galaxies. We use the measurements at $4.85 \mathrm{GHz}$ because they are our most accurate. The 4 galaxies that have the highest absolute radio excesses are located at projected distances smaller than 2 degrees $(\sim 0.6 \mathrm{Mpc})$ and are labeled in Fig. 5. All four galaxies are classified as centrally active (VCC0836 (NGC 4388): Sy2, VCC1043 (NGC 4388): Sy/LINER, VCC1632 (NGC 4552): LINER/HII, VCC1727 (NGC 4579): Sy2/LINER). In addition, VCC0836, VCC1043 and VCC1727 show extended radio emission. In the case of VCC1043 the radio emission, which is not due to a jet, is even extraplanar (Kotanyi et al. 1983). But as can be seen in Fig. 4e, when one subtracts the flux density of the FIRST central point sources, we no longer observe a radio excess. We have also calculated the relative radio excess, i.e. the absolute radio excess divided by the expected radio flux density calculated using the radio-FIR correlation. All four galaxies cited above have a relative radio excess greater than 2 . This is also the case for VCC0222 (NGC 4235, Sy1) and VCC1978. Thus, only galaxies that host an active center show a radio excess. 


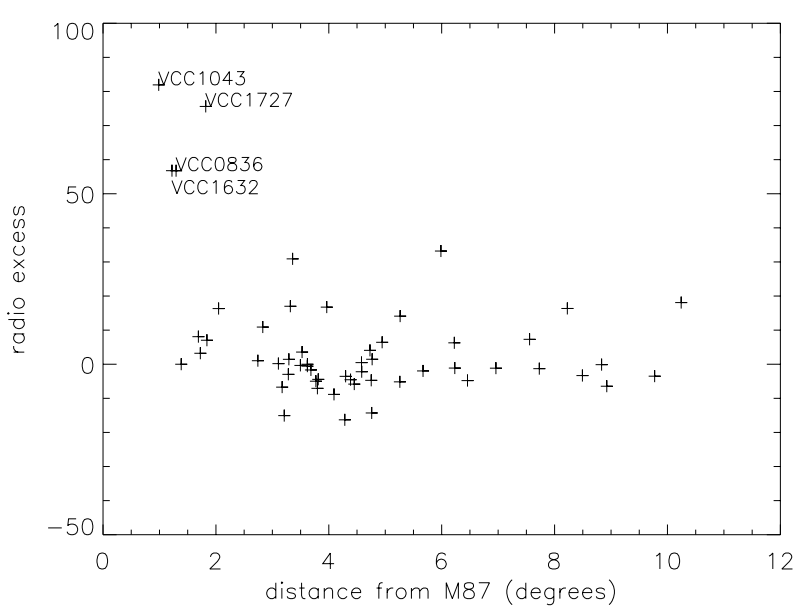

Fig. 5. Radio excess over the radio-FIR correlation of the observed VCC galaxies as a function of the projected distance to the cluster center (M 87).

\section{The spectral index distribution}

The distribution of the spectral index of the Virgo galaxies as a function of the projected distance to the cluster center (M 87) can be seen in Fig. 6. Elliptical galaxies are represented as triangles. The galaxies with a spectral index greater than -0.5 are labeled. They are all spiral or S0 galaxies. VCC0222 is classified as a Sy1, VCC1110 as a LINER, and VCC1727 as a Sy2. The spectral index of VCC1200 has to be taken with some caution, because the $8.6 \mathrm{GHz}$ flux density might be overestimated. VCC1450, VCC1535 and VCC1987 do not show any particular central activity. VCC1987 (NGC 4654) has most probably undergone a tidal interaction and is experiencing ram pressure now (Vollmer 2003). Moreover, it shows an off-center molecular bar (Sofue et al. 2003). One could speculate that it is just developing a central activity. The galaxies with a spectral index $>-0.5$ are not concentrated towards the cluster center. Thus there is no indication that cluster environment leads to flatter spectral indices via central activity nor to steeper spectra as expected from better confinement of relativistic electrons. There might be a weak trend that the dispersion of the spectral index increases with decreasing projected distance to the cluster center. The steepest spectral indices $\alpha<-1.0$ are found in $\mathrm{Sc} / \mathrm{Scd}$ galaxies and the elliptical galaxy VCC0763.

The number distribution of the spectral index is shown in Fig. 7. The dashed line traces the distribution of all sample galaxies, the solid line that of the Virgo cluster members. Both distributions peak around a spectral index of $\alpha=-0.9$. Both distributions show a tail towards larger spectral indices than the peak value. The steepest spectra $\alpha<-1.2$ are found outside the Virgo cluster.

\section{Discussion and conclusions}

We confirm the finding of Gavazzi \& Boselli (1999b) that Virgo cluster galaxies statistically do not show an enhanced radio emission compared to isolated galaxies. However, a few spiral galaxies show an excess in the absolute radio/FIR ratio. These galaxies all possess active centers (LINER, HII, AGN) and are

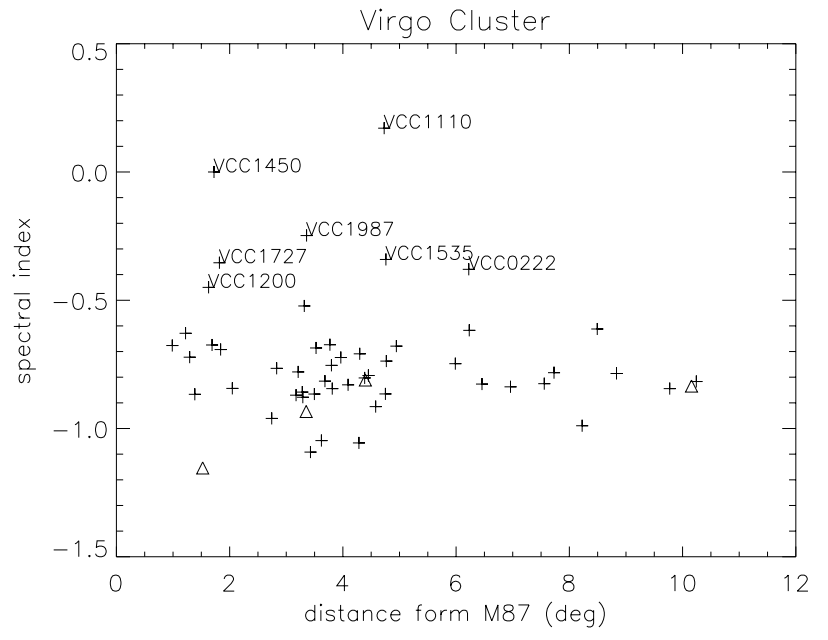

Fig. 6. Spectral index of the Virgo galaxies as a function of the projected distance to the cluster center (M 87).

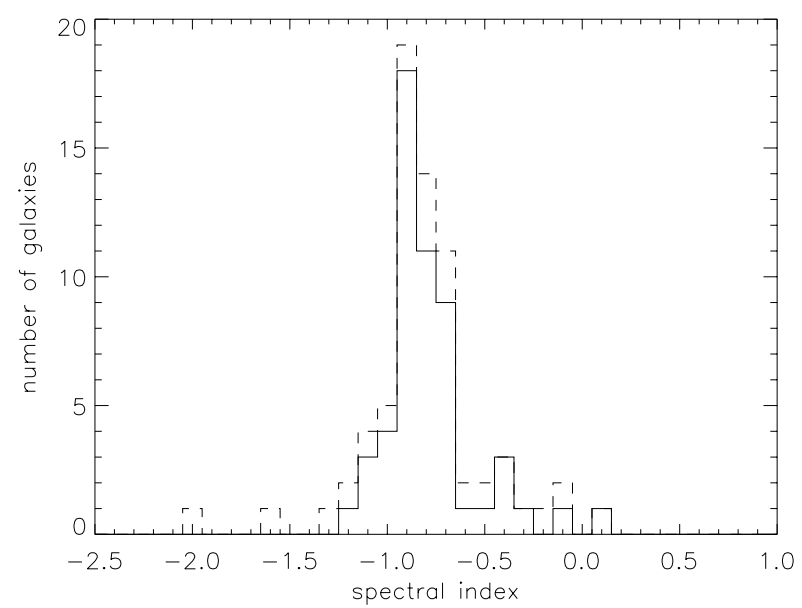

Fig. 7. Number distribution of the spectral index. Solid line: Virgo galaxies. Dashed line: all sample galaxies.

located within a radius of $2^{\circ}$ from the cluster center. We confirm the conclusion of Niklas et al. (1995) based on a small number of galaxies that the spectral index of Virgo galaxies does not change significantly with projected cluster distance. There might be a trend that the scatter of the spectral indices increases with decreasing projected distance. The change in the behaviour of radio sources within the Virgo cluster (radio excess and spectral index) is due to central activity. It is not clear if and under what conditions the cluster environment triggers central activity.

As described in Sect. 1, during the phase of active ram pressure stripping, the magnetic field is compressed and one expects an excess of radio emission with respect to the FIR emission. This extended radio emission should be asymmetric in the outer parts of the disk. This seems not to be the case in the Virgo cluster. Based on the HI observations of the brightest spiral galaxies (Cayatte et al. 1990), no clear case of active ram pressure stripping could be identified. The only possible case is VCC1043 (NGC 4438), but there the galaxy had also 
a strong tidal interaction (Combes et al. 1988). A clearer case is the edge-on galaxy VCC1516 (NGC 4522) where high column density extraplanar HI is detected (Kenney et al. 2003, in prep.). Neither galaxy shows an enhanced, asymmetric radio continuum emission. However, in VCC1516 the compression is visible as a region of enhanced polarization. In barred galaxies the situation is similar. Whereas in the compression upstream regions of the bar the radio polarization is enhanced, an excess of total radio continuum emission is not observed (Beck et al. 2001). We might speculate that this is the case, because both galaxies are stripped almost face-on, in which case the asymmetric compression is minimum. Other possibilities are: (i) the turbulent magnetic field is enhanced via an enhanced star formation rate due to compression. The enhancement of the ordered magnetic field due to compression might then be negligible with respect to the turbulent magnetic field, but it can still be observed in polarization. (ii) During compression the scale height of the magnetic field increases, which leads to a decrease of the total magnetic field strength.

In the Coma cluster the situation is different. HI observations of the brightest spiral galaxies revealed highly asymmetric gas disks of high column density (Bravo-Alfaro et al. 2000). At the same time, Gavazzi \& Boselli (1999b) found a statistical excess of radio emissivity for Coma cluster galaxies. This is a puzzling result, because the Virgo cluster is dynamical young (asymmetric galaxy distribution, constant velocity distribution of spirals), whereas the Coma cluster is more relaxed. One would thus expect that ram pressure is more active in the Virgo cluster. The solution to this paradigm is that the core radius of the Virgo cluster ICM distribution is much smaller than that of the Coma cluster. Thus Virgo galaxies have to come very close to the cluster center to be significantly stripped (Vollmer et al. 2001). In this case the phase of active ram pressure stripping is much shorter in the Virgo cluster than in the Coma cluster due to the small ICM core radius and the high galaxy velocities near the cluster center.
Acknowledgements. This work is based on observations with the 100$\mathrm{m}$ telescope of the MPIfR (Max-Planck-Institut für Radioastronomie) at Effelsberg.

\section{References}

Beck, R., Shoutenkov, V., Ehle, M., et al. 2002, A\&A, 391, 83

Bothun, G., Schommer, R. A., \& Sullivan, W. T. III 1982, AJ, 87, 731

Cayatte, V., van Gorkom, J. H., Balkowski, C., \& Kotanyi, C. 1990, AJ, 100, 604

Cayatte, V., Kotanyi, C., Balkowski, C., \& van Gorkom, J. H. 1994, AJ, 107, 1003

Chamaraux, P., Balkowski, C., \& Gérard, E. 1980, A\&A, 83, 38

Combes, F., Dupraz, C., Casoli, F., \& Pagani, L. 1988, A\&A, 203, L9

Condon, J. J., Cotton, W. D., Greisen, E. W., et al. 1998, AJ, 115, 1693

Binggeli, B., Sandage, A., \& Tammann, G. 1985, AJ, 90, 1681 (VCC)

Bravo-Alfaro, H., Cayatte, V., van Gorkom, J. H., \& Balkowski, C. 2000, AJ, 119, 580

de Jong, T., Klein, U., Wielebinski, R., \& Wunderlich, E. 1985, A\&A, 147, L6

Gavazzi, G. 1987, ApJ, 320, 96

Gavazzi, G. 1989, ApJ, 346, 59

Gavazzi, G., \& Boselli, A. 1999a, A\&A, 343, 86

Gavazzi, G., \& Boselli, A. 1999b, A\&A, 343, 93

Gavazzi, G., Boselli, A., Donati, A., Franzetti, P., \& Scodeggio, M. 2003, A\&A, 400, 451

Giovanelli, R., \& Haynes, M. P. 1985, ApJ, 292, 404

Gunn, J. E., \& Gott, J. R. 1972, ApJ, 176, 1

Kotanyi, C., van Gorkom, J. H., \& Ekers, R. D. 1983, ApJ, 273, L7

Niklas, S., Klein, U., \& Wielebinski, R. 1995, A\&A, 293, 56

Niklas, S. 1997, A\&A, 322, 29

Otmianowska-Mazur, K., \& Vollmer, B. 2003, A\&A, 402, 879

Ott, M., Witzel, A., Quirrenbach, A., et al. 1994, A\&A, 284, 331

Sofue, Y., Koda, J., Nakanishi, H., et al. 2003, PASJ, 55, 17

Vollmer, B., Cayatte, V., Balkowski, C., \& Duschl, W. J. 2001, ApJ, 561, 708

Vollmer, B. 2003, A\&A, 398, 525

Wunderlich, E., Wielebinski, R., \& Klein, U. 1987, A\&A, 69, 487 


\section{Online Material}


B. Vollmer et al.: Virgo radio continuum spectra, Online Material p 2
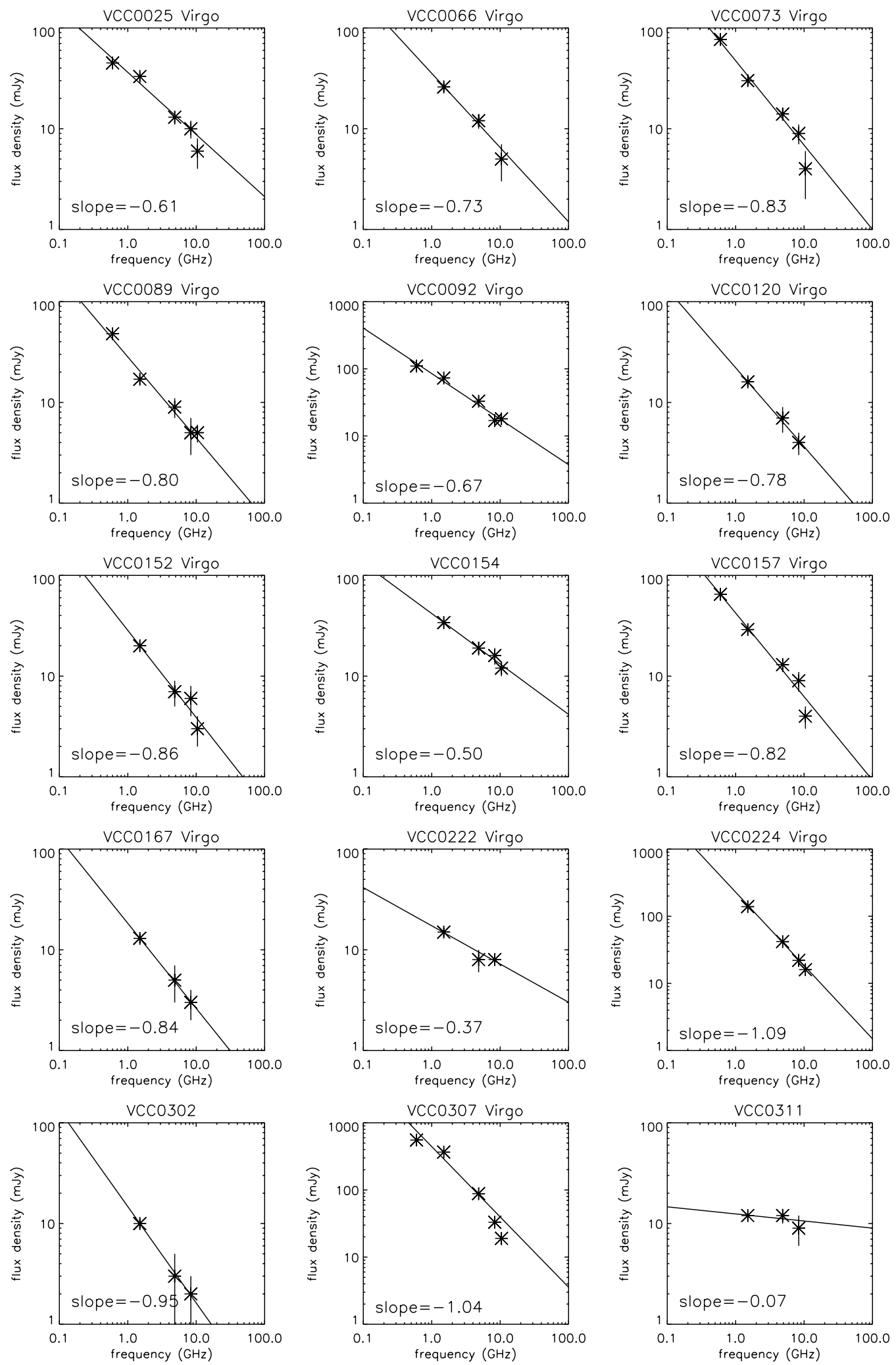

Fig. 3. Radio spectra of the observed VCC sources. 
B. Vollmer et al.: Virgo radio continuum spectra, Online Material p 3
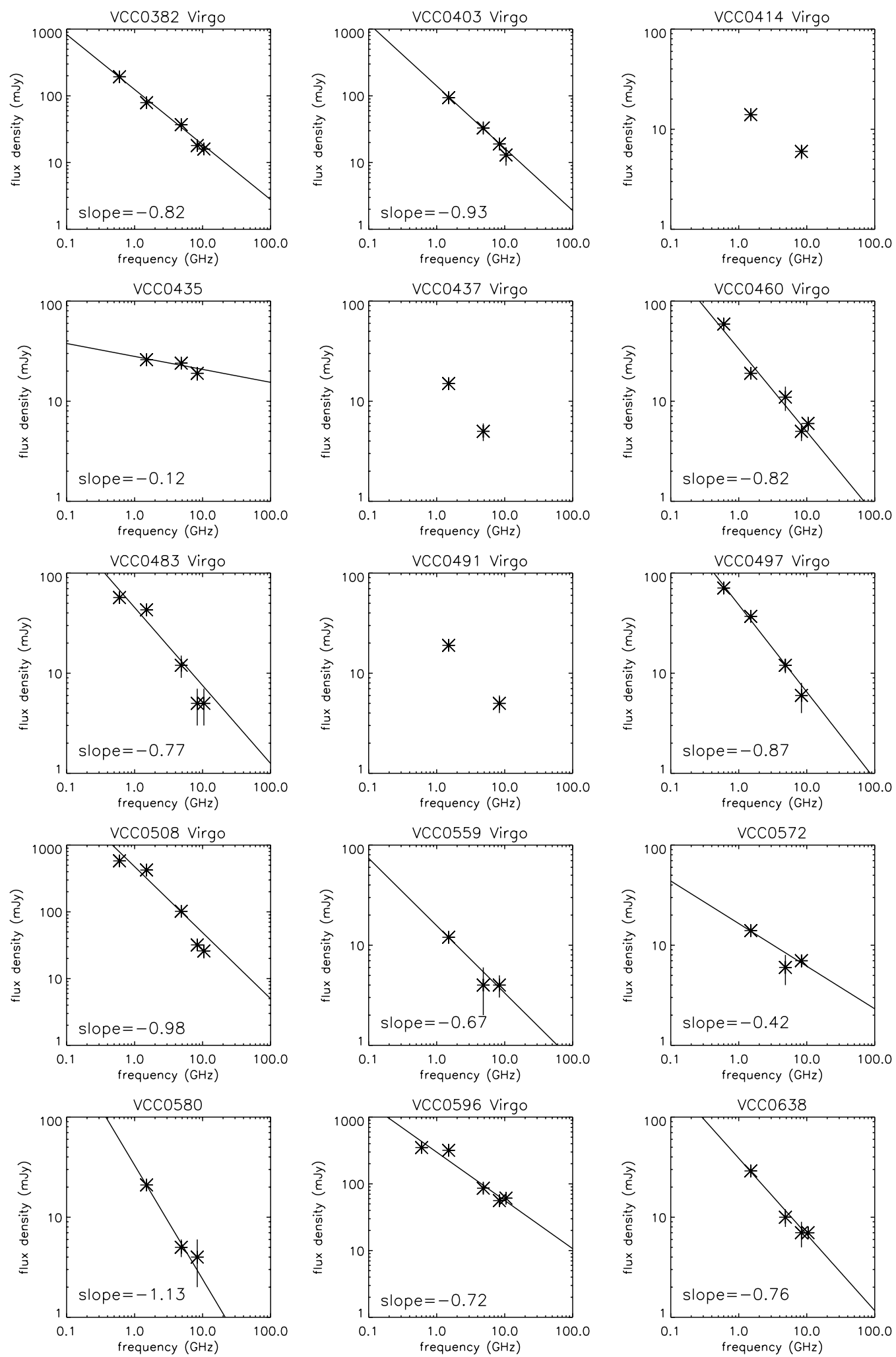

Fig. 3. continued. 
B. Vollmer et al.: Virgo radio continuum spectra, Online Material p 4
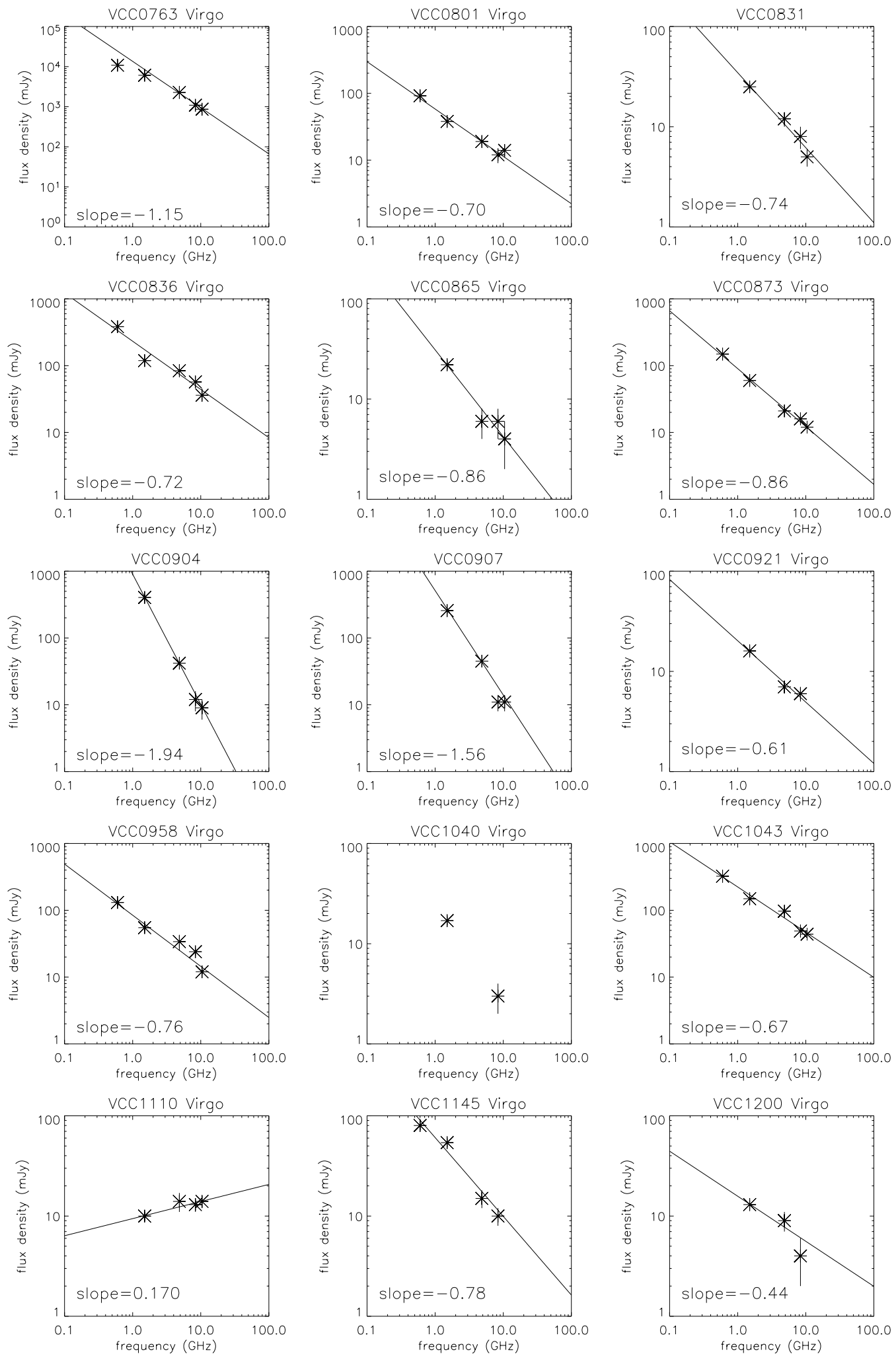

Fig. 3. continued. 
B. Vollmer et al.: Virgo radio continuum spectra, Online Material p 5
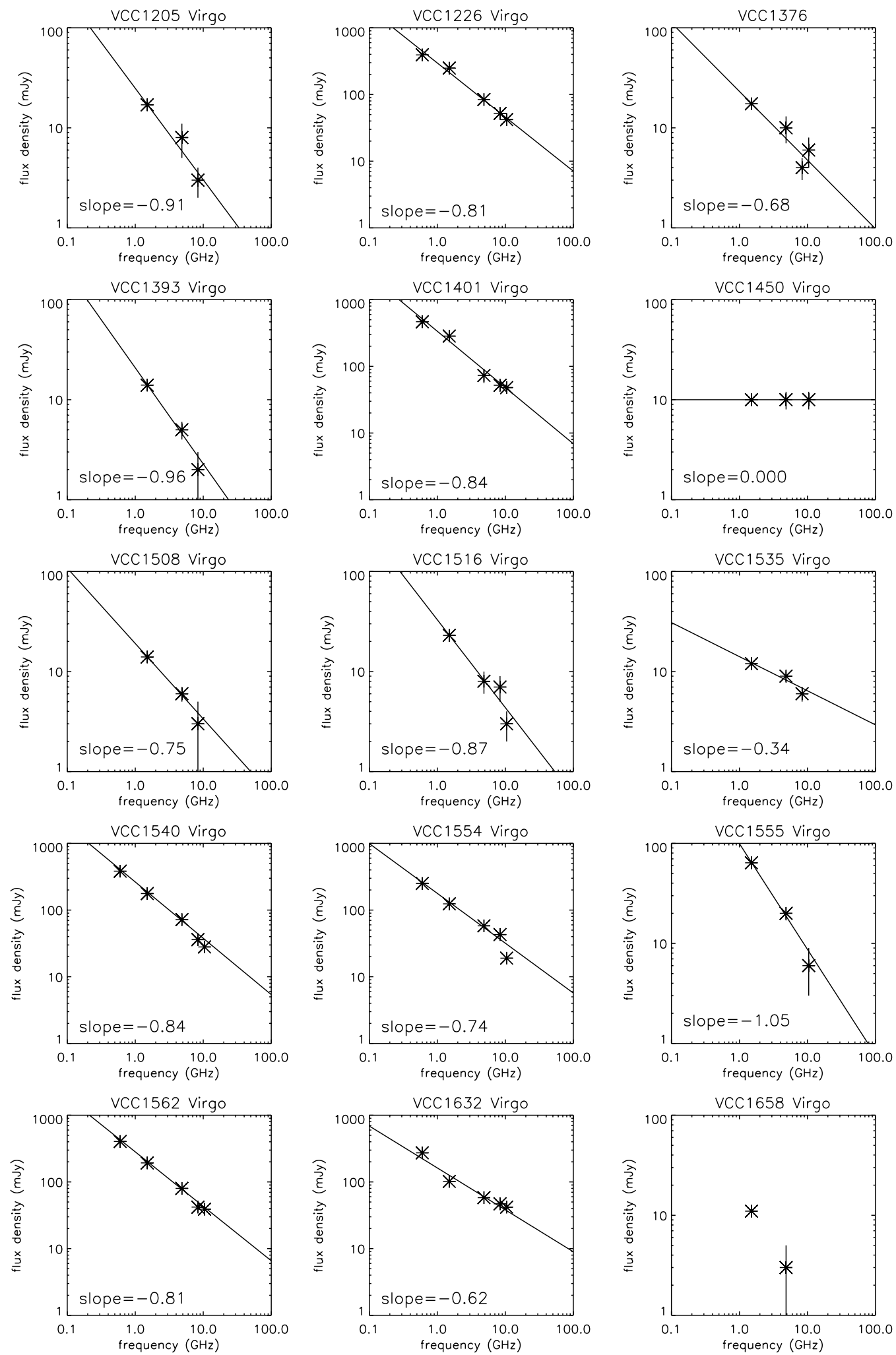

Fig. 3. continued. 
B. Vollmer et al.: Virgo radio continuum spectra, Online Material p 6
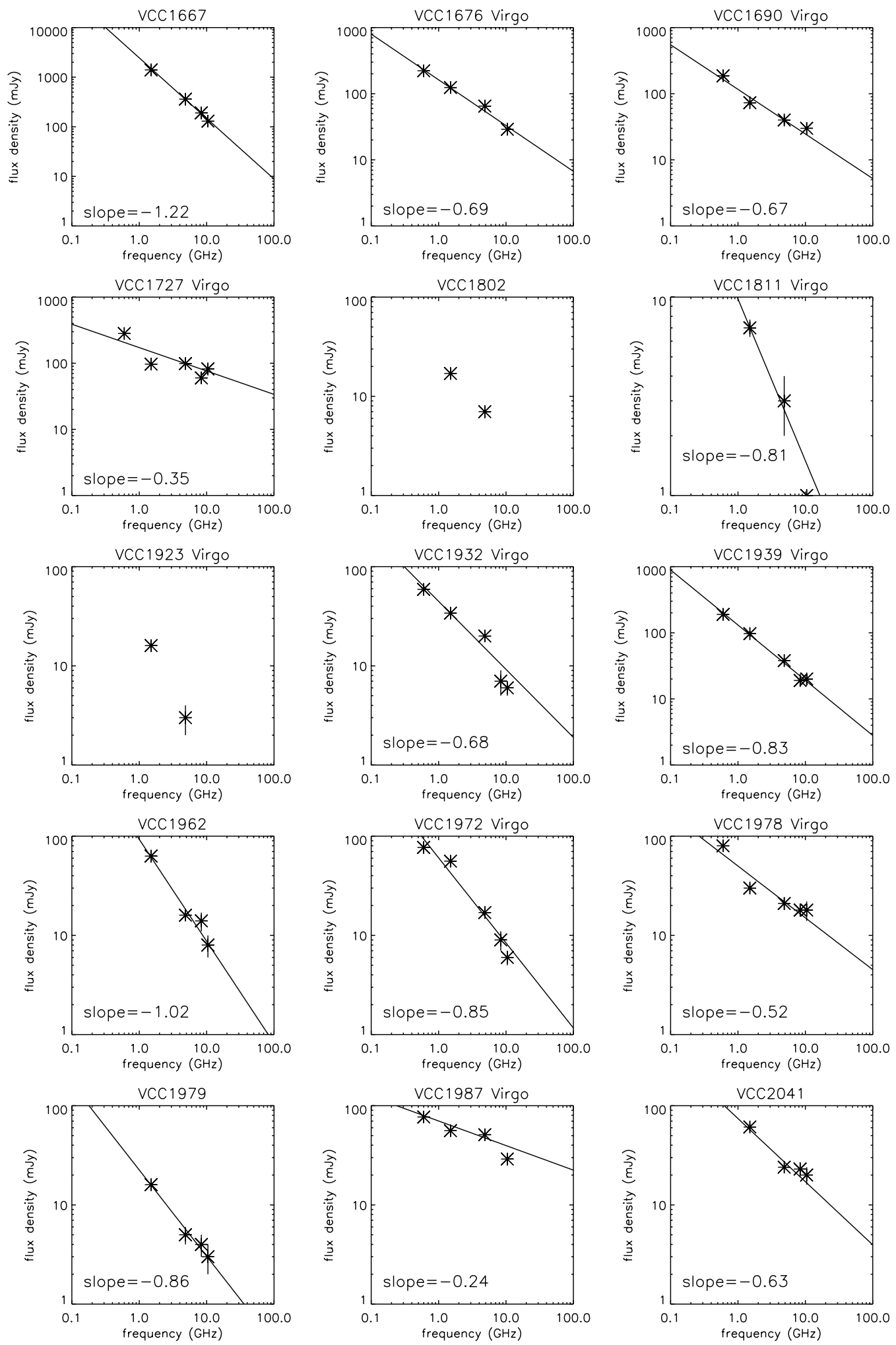

Fig. 3. continued. 
B. Vollmer et al.: Virgo radio continuum spectra, Online Material p 7
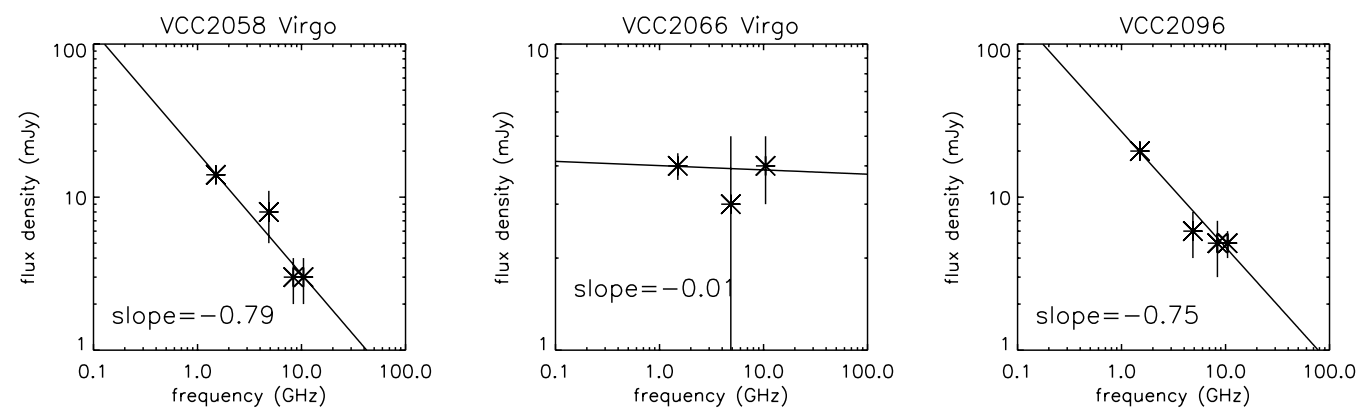

Fig. 3. continued. 